\title{
Mott-Hubbard transition and spin-liquid state on the pyrochlore lattice
}

\author{
Nyayabanta Swain, ${ }^{1,2}$ Rajarshi Tiwari, ${ }^{3}$ and Pinaki Majumdar ${ }^{1,2}$ \\ ${ }^{1}$ Harish-Chandra Research Institute, Chhatnag Road, Jhusi, Allahabad 211019, India \\ ${ }^{2}$ Homi Bhabha National Institute, Training School Complex, Anushakti Nagar, Mumbai 400085, India \\ ${ }^{3}$ School of Physics and CRANN, Trinity College, Dublin 2, Ireland
}

(Received 28 July 2016; published 13 October 2016)

\begin{abstract}
The pyrochlore lattice involves corner-sharing tetrahedra and the resulting geometric frustration is believed to suppress any antiferromagnetic order for Mott insulators on this structure. There are nevertheless short-range correlations which could be vital near the Mott-Hubbard insulator-metal transition. We use a static auxiliaryfield-based Monte Carlo to study this problem in real space on reasonably large lattices. The method reduces to unrestricted Hartree-Fock at zero temperature but captures the key magnetic fluctuations at finite temperature. Our results reveal that increasing interaction drives the nonmagnetic (semi) metal to a "spin disordered" metal with small local moments, at some critical coupling, and then, through a small pseudogap window, to a large moment, gapped, Mott insulating phase at a larger coupling. The spin disordered metal has a finite residual resistivity which grows with interaction strength, diverging at the upper coupling. We present the resistivity, optical conductivity, and density of states across the metal-insulator transition and for varying temperature. These results set the stage for the more complex cases of Mott transition in the pyrochlore iridates and molybdates.
\end{abstract}

DOI: 10.1103/PhysRevB.94.155119

\section{INTRODUCTION}

The presence of geometric frustration disfavors long-range antiferromagnetic order and promotes a complex magnetic state in insulating magnets with short-range interaction [1,2]. Two complications arise in magnetic insulators close to a Mott insulator-metal transition (IMT): (i) the "virtual hopping" of the electrons mediate long-range and multispin coupling, and (ii) the magnitude of the local moments weaken as the system is pushed towards the IMT. The first effect can lift the degeneracy of the short-range model and promote an ordered state while the second tends to destroy the magnetic state altogether. The outcome of this interplay is lattice specific [3,4], of relevance to several real life materials, and requires tools beyond those usually applied in frustrated magnetism.

The possibilities in charge transport are also interesting. While the large moment, gapped, Mott phase is insulating, the strong suppression of the moment near the IMT, and possible orientational randomness due to frustration, can generate a "bad metal" state on the disordered magnetic background. Such a state can involve a pseudogap, an unusually large lowtemperature resistivity [5-7], and, possibly, an anomalous Hall response if the moments organize in a noncoplanar manner [8].

The pyrochlores are a fascinating structure [9] to explore these effects. In the deep Mott state on a pyrochlore lattice, where one expects only nearest-neighbor antiferromagnetic coupling, the effective model can be written as a sum, over the tetrahedra, of squares of the total moment in each tetrahedron $[10,11]$. The minimum of this is infinitely degenerate since the four spins at the vertices of each tetrahedron just need to satisfy a zero vector sum. The appearance of longer-range couplings as the electron-electron Hubbard repulsion reduces (or the bandwidth increases) can, potentially, lift the degeneracy and promote some ordered state. The transport and spectral character between the spin-liquid Mott insulator and the band semimetal is also not known.

There are, remarkably, experimental realizations of Mott physics on the pyrochlore structure, as observed in the rare-earth molybdates [12-17] and iridates [18-21]. These, however, involve $4 d$ or $5 d$ electrons and require Hund's coupling or spin-orbit interaction, in addition to the Hubbard interaction, for a successful description. To set the stage for these more realistic but complex models, this paper focuses on the role of just the Hubbard interaction on the half-filled pyrochlore lattice. We address two broad questions: (i) What is the nature of the magnetic state as one moves towards weaker interaction in the Mott insulator, and in particular what are the magnetic correlations near the IMT? (ii) What is the impact of these magnetic correlations on electron physics (resistivity, optics, spectral features) near the IMT?

Our main results, obtained by using a real-space static auxiliary-field-based method, are the following: (i) Increasing interaction in the ground state leads successively to a smallmoment metal, then a narrow insulating pseudogap window, and finally to the gapped Mott insulator. (ii) The resistivity and the low energy density of states have a strongly nonmonotonic temperature dependence near the metal-insulator transition (MIT). (iii) The finite-moment phases, from near the MIT to the deep Mott regime, are all disordered. Well into the Mott phase they display what seems (within the limits of our system size) to be power-law spatial correlations that survive to a small finite temperature.

The paper is organized as follows: In the next section we provide a summary of existing results on the pyrochlore structure - in particular the Heisenberg and Hubbard models. This is followed by a description of our method. The next section presents results on the phase diagram, density of states, transport, and optics, across the MIT. The final section expands on a few issues like effective models for the magnetism, computational issues, and the magnetic structure factor.

\section{PREVIOUS WORK}

The pyrochlore Heisenberg antiferromagnet model, which describes the deep Mott limit, has been well studied, the 
Hubbard model much less so. We summarize the key results from these and then move to our method and results.

\section{A. Pyrochlore Heisenberg model}

While the Hubbard model at half filling and large interaction maps on to the $S=1 / 2$ Heisenberg antiferromagnet, we provide a more general discussion of this model below.

The nearest-neighbor classical Heisenberg antiferromagnet, $H=J \sum_{\langle i j\rangle} \mathbf{S}_{i} \cdot \mathbf{S}_{j}, J>0$, on the pyrochlore lattice does not show any magnetic order down to zero temperature $[10,11]$ $(T=0)$. The model can be written as $H \equiv(J / 2) \sum_{\alpha} \mathbf{P}_{\alpha}^{2}$, up to a constant, where $\mathbf{P}_{\alpha}=\sum_{i=1}^{4} \mathbf{S}_{i}$, where the $\mathbf{S}_{i}$ are spins on the tetrahedron $\alpha$. The ground state needs to satisfy the constraint $\mathbf{P}_{\alpha}=0$ for all $\alpha$. This results in an infinitely degenerate manifold of possible configurations $\left\{\mathbf{S}_{i}\right\}$. It has been established that there are no internal energy barriers between these degenerate minima at $T=0$, and no free-energy barriers at finite $T$, precluding a freezing transition either [11]. Since the moments are corner-shared between tetrahedra, the constraints lead to a correlated spin structure. The "disordered" but long-range correlated state generates characteristic features in the magnetic structure factor, appearing as "bow ties" [22] or "pinch points" [23], indicative of power-law correlations [23,24].

In the semiclassical $S \gg 1$ case the classical degeneracy is partially lifted by the zero-point energy of quantum fluctuations at harmonic order, but there remains an infinite manifold of degenerate collinear ground states [25]. Further quantum fluctuations at anharmonic order break the degeneracy between the various harmonic ground states, yet they leave out a massive but nonextensive degeneracy (smaller than the harmonic ground state) [26].

The ground state for $S=1 / 2$ is argued to be a quantum spin liquid [27-29]. This spin-singlet ground state has a finite energy gap for triplet excitations. The spin-spin correlation function decays exponentially with distance with a correlation length shorter than the lattice spacing [27].

\section{B. Pyrochlore Hubbard model}

Heisenberg interactions beyond nearest neighbor induce transitions to various ordered phases (e.g., collinear, nematic, and multiple-q order) [30-32]. Also, easy axis anisotropy, long-range dipolar interaction, etc., lead to multiple-q ordered phases [33]. It has been argued that, beyond the Heisenberg limit, the half-filled Hubbard model on the pyrochlore lattice can be expressed as a highly frustrated intratetrahedral spin model with weak intertetrahedral perturbations [29]. This model has an exactly solvable Klein point, about which the ground state is a three-dimensional quantum spin liquid over an extended parameter region. This spin-liquid state hosts massive spinon excitations, which are deconfined and move in all three dimensions within the lattice [29]. For the Hubbard model on the pyrochlore lattice, the only work on the Mott transition that we know of suggests a transition from a semimetal to a spin-liquid Mott insulator [34]. However, detailed properties near the IMT are not available.

\section{Hubbard with additional interactions}

In the pyrochlore iridates $R_{2} \mathrm{Ir}_{2} \mathrm{O}_{7}$, both the $R$ (rare earth or $\mathrm{Y}$ ) and the Ir live in two interpenetrating pyrochlore structures. Their physics is mainly dictated by the $5 d$ electrons of Ir, which have strong spin-orbit coupling and moderate Hubbard repulsion $U$ (due to large spatial extent of $5 d$ orbitals). This strong spin-orbit coupling lifts the orbital degeneracy of $5 d$ electrons, reduces the bandwidth, and leads to an effective single-band description in terms of pseudospin $j_{\text {eff }}=1 / 2$ states. In the absence of $U$, the ground state is a semimetal or topological insulator depending on the ratio of spin-orbit coupling and hopping [35] $(t)$. This picture remains unchanged for weak $U / t$. For strong $U / t$, a Hartree-Fock calculation shows the system becoming an "all-in-all-out" magnetic insulator. Near the magnetic transition, a topological Weyl semimetal phase shows up [35]. A more elaborate cluster dynamical mean-field theory (C-DMFT) calculation confirms this scenario [36].

In the pyrochlore molybdates $R_{2} \mathrm{Mo}_{2} \mathrm{O}_{7}$, the physics is mainly governed by the $4 d$ electrons of Mo. The presence of a trigonal crystal field and strong Hund's coupling leads to band narrowing [37]. Electron correlation then acts on a background involving double exchange ferromagnetism competing with superexchange antiferromagnetism. A Hartree-Fock calculation shows the possible spin and orbital order in the ground state for the molybdates [38].

\section{MODEL AND METHOD}

We study the single-band Hubbard model, with nearestneighbor hopping, on the pyrochlore lattice:

$$
H=H_{0}+U \sum_{i} n_{i \uparrow} n_{i \downarrow},
$$

where $H_{0}=\sum_{i j, \sigma}\left(t_{i j}-\mu \delta_{i j}\right) c_{i \sigma}^{\dagger} c_{j \sigma}$. The $t_{i j}=-t$ for nearest-neighbor hopping on the pyrochlore lattice and $U>0$ is the Hubbard repulsion. We will set $t=1$. The chemical potential $\mu$ is varied to maintain the density at $n=1$ as the interaction and temperature $T$ are varied.

We use a Hubbard-Stratonovich (HS) transformation [39] that introduces a vector field $\mathbf{m}_{i}(\tau)$ and a scalar field $\phi_{i}(\tau)$ at each site to decouple the interaction. This decomposition $[40,41]$ retains the rotation invariance of the Hubbard model, and hence the correct low-energy excitations, and reproduces unrestricted Hartree-Fock theory at $T=0$.

We treat the $\mathbf{m}_{i}$ and $\phi_{i}$ as classical fields, i.e., neglect their time dependence, but completely retain the thermal fluctuations in $\mathbf{m}_{i} . \phi_{i}$ is treated at the saddle-point level, i.e., $\phi_{i} \rightarrow\left\langle\phi_{i}\right\rangle=(U / 2)\left\langle\left\langle n_{i}\right\rangle\right\rangle=U / 2$ at half filling, since charge fluctuations would be penalised at temperatures $T \ll U$. Retaining the spatial fluctuations of $\mathbf{m}_{i}$ allows us to estimate $T_{c}$ scales and access the crucial thermal effects on transport. In the literature the overall scheme is known as the "static path approximation" (SPA) to the functional integral for the partition function $[42,43]$. We have used it in the past to address the Mott transition on the triangular [44] lattice. Others have used it successfully in superconductors [43], etc. We will discuss the limitations of this scheme later in the paper.

Within this approach the half-filled Hubbard problem is mapped onto electrons coupled to the field $\mathbf{m}_{i}$, which itself 
follows a distribution function $P\left\{\mathbf{m}_{i}\right\}$ :

$$
\begin{aligned}
H_{\mathrm{eff}} & =H_{0}-\frac{U}{2} \sum_{i} \mathbf{m}_{i} \cdot \vec{\sigma}_{i}+\frac{U}{4} \sum_{i} \mathbf{m}_{i}^{2}, \\
P\left\{\mathbf{m}_{i}\right\} & \propto \operatorname{Tr}_{c c^{\dagger}} e^{-\beta H_{\mathrm{eff}}},
\end{aligned}
$$

where the chemical potential $\mu$ in $H_{0}$ is replaced by $\tilde{\mu}=\mu-$ $\frac{U}{2}$. $H_{\text {eff }}$ can be seen as comprising of an electronic Hamiltonian, $H_{\text {el }}$ (the first two terms) and the classical "stiffness" $H_{c l}=$ $\frac{U}{4} \sum_{i} \mathbf{m}_{i}^{2}$. In an exact calculation, where the dynamics of $\mathbf{m}_{i}$ and $\phi_{i}$ are retained, $H_{\text {eff }}$ would be replaced by an effective action while $P$ would be replaced by a fermion determinant in the $\{\mathbf{m}, \phi\}$ background.

Within SPA $H_{\text {eff }}$ and $P\left\{\mathbf{m}_{i}\right\}$ define a coupled fermionlocal-moment problem. If the moments are large and random the electronic problem requires numerical diagonalization. Similarly, the $P\left\{\mathbf{m}_{i}\right\}$ cannot be written down in closed form since the fermion free energy is not known for arbitrary $\left\{\mathbf{m}_{i}\right\}$ background. The method of choice in these situations is a combination of Monte Carlo (MC) for updating the $\mathbf{m}_{i}$ with exact diagonalization (ED) of the fermion Hamiltonian for computing the Metropolis update cost.

To access large sizes in limited time, we use a cluster algorithm for estimating the update cost. The energy cost of updating the variable $\mathbf{m}_{i}$ is computed by diagonalizing a cluster (of size $N_{c}$, say) constructed around the site $\mathbf{R}_{i}$. We have extensively benchmarked this method [45]. Results in this paper are obtained for lattices of $6^{3}$ unit cells with four atoms per unit cell, using a cluster with $3^{3} \times 4$ atoms.

Electronic properties are calculated by diagonalizing $H_{\mathrm{el}}$ on the full lattice for equilibrium $\left\{\mathbf{m}_{i}\right\}$ configurations. From the equilibrium configurations we compute the single-particle density of states (DOS) as $N(\omega)=\frac{1}{2 N}\left\langle\sum_{n} \delta\left(\omega-\epsilon_{n}\right)\right\rangle$ where $\langle\ldots\rangle$ indicates thermal average over equilibrium configurations. For a $L \times L \times L$ pyrochlore lattice, the total number of lattice sites $N=4 L^{3}$. We calculate the optical conductivity by using the Kubo formula [46] as follows:

$$
\sigma^{x x}(\omega)=\frac{\sigma_{0}}{N} \sum_{n, m} \frac{f\left(\epsilon_{n}\right)-f\left(\epsilon_{m}\right)}{\epsilon_{m}-\epsilon_{n}}\left|J_{x}^{n m}\right|^{2} \delta\left(\omega-\left(\epsilon_{m}-\epsilon_{n}\right)\right),
$$

where $J_{x}^{n m}=\left\langle n\left|J_{x}\right| m\right\rangle$ and $J_{x}$ is the current operator given by

$$
J_{x}=-i \sum_{i, \sigma}\left[\left(t c_{i, \sigma}^{\dagger} c_{i+\hat{x}, \sigma}-\text { H.c. }\right)\right] \text {, }
$$

$f\left(\epsilon_{n}\right)$ is the Fermi function, and $\epsilon_{n}$ and $|n\rangle$ are the singleparticle eigenvalues and eigenstates of $H_{\mathrm{el}}\left\{\mathbf{m}_{i}\right\}$, respectively. The conductivity is in units of $\sigma_{0}=e^{2} /\left(\hbar a_{0}\right)$, where $a_{0}$ is the lattice constant. The dc conductivity is obtained as a low-frequency average of the optical conductivity over a window $\sim 0.05 t$. We calculate average local magnetic moment $m_{\mathrm{avg}}=\frac{1}{N} \sum_{i}^{N}\left\langle\left|\mathbf{m}_{i}\right|\right\rangle$ and their distribution $P(m)=$ $\frac{1}{N}\left\langle\sum_{i} \delta\left(m-\left|\mathbf{m}_{\mathbf{i}}\right|\right)\right\rangle$ from the equilibrium configurations. The magnetic structure factor is computed as $S(\mathbf{q})=\frac{1}{N^{2}} \sum_{i j}\left\langle\mathbf{m}_{i}\right.$. $\left.\mathbf{m}_{j}\right\rangle e^{i \mathbf{q} \cdot\left(\mathbf{r}_{i}-\mathbf{r}_{j}\right)}$ at each temperature. The rapid growth of a mode at some $\mathbf{q}=\mathbf{Q}$ (say) indicates the onset of magnetic order. Our results are averaged over 100 equilibrium Monte Carlo configurations.
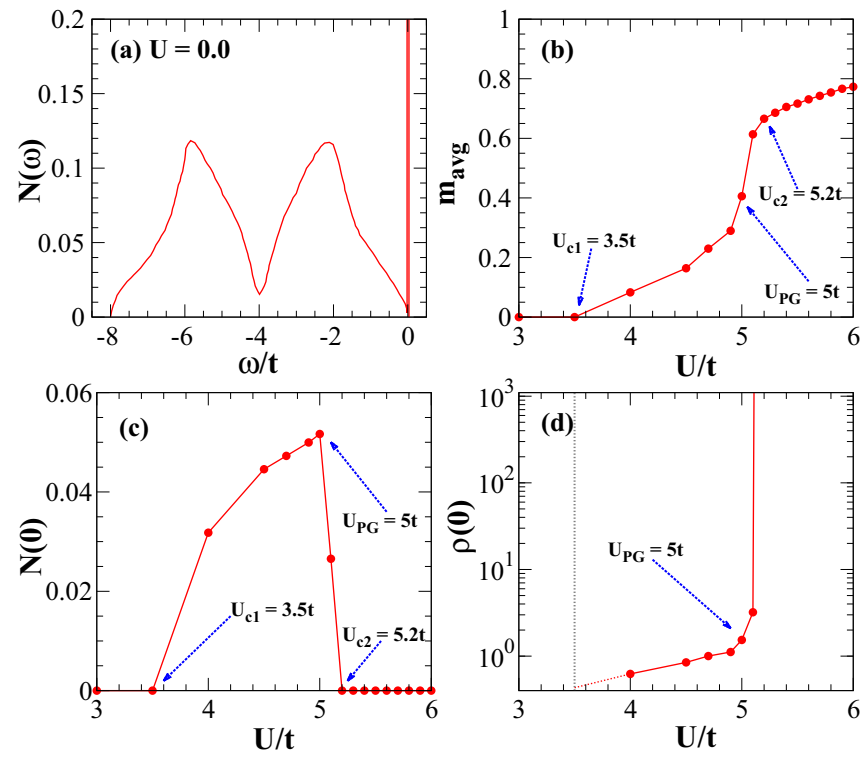

FIG. 1. (a) Tight-binding density of states for the pyrochlore lattice. Results at $T=0$ for (b) variation of the average local moment $m_{\text {avg }}$, (c) the density of states $N(0)$ at the Fermi level, and (d) the resistivity $\rho(T=0)$.

\section{RESULTS}

\section{A. The ground state}

As $T \rightarrow 0$ our MC mainly samples configurations that maximize $P\left\{\mathbf{m}_{i}\right\}$ or, alternately, minimize the energy, i.e., $\frac{\delta}{\delta \mathbf{m}_{i}}\left\langle H_{\text {eff }}\right\rangle=0$. This is the same as unrestricted Hartree-Fock in the magnetic channel.

Up to a critical coupling $U_{c 1} \sim 3.5 t$ the minimization yields $m_{i}=\left|\mathbf{m}_{i}\right|=0$ at all sites. As a result up to $U_{c 1}$ the electronic ground state is essentially tight binding, the density of states for which is shown in Fig. 1(a). There is a sharply suppressed DOS at the Fermi level characteristic of the pyrochlore band structure, and a flat band right above the Fermi level. For $U<U_{c 1}$ the system is a semimetal.

For $U_{c 1}<U<U_{P G}$ where $U_{P G} \approx 5 t$ we observe a smallmoment, orientationally disordered, magnetic state. The average moment size is shown in Fig. 1(b), and the full distribution later in the paper. With the disorder caused by these moments breaking the translation invariance of the pyrochlore lattice, the DOS at the Fermi level, $N(0)=\int_{-\Omega}^{\Omega} N(\omega) d \omega / \int_{-\Omega}^{\Omega} d \omega$, where $\Omega=0.05 t$, gains weight [Fig. 1(c)]. In a narrow region around $U_{P G}$ there is rapid increase in the mean magnitude of the moments and, as a result, the DOS at the Fermi level gets depressed again. The detailed behavior of the low-energy DOS is shown later. At $U_{c 2} \sim 5.2 t$ the DOS at the Fermi level vanishes as a Mott gap opens. For $U>U_{c 2}$ the moments are large and saturate to their atomic value, $\left|\mathbf{m}_{i}\right|=1$, as $U / t \rightarrow$ $\infty$. The coupling of the electrons to the local moments leads to weak scattering and a small resistivity for $U_{c 1}<U<U_{P G}$, a rapid growth in resistivity for $U_{P G}<U<U_{c 2}$, and zero dc conductivity for $U>U_{c 2}$. This is shown in Fig. 1(d). We will discuss the resistivity in much greater detail later and just wanted to highlight the effect within the $T=0$ mean-field state here. 

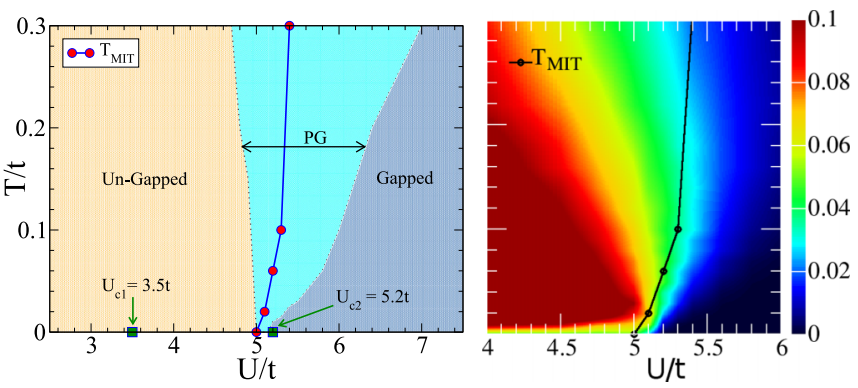

FIG. 2. Phase diagram (left) and the density of states at the Fermi level (right) for varying $U$ and $T$. Local moments appear at $U_{c 1}$ but the state remains metallic, turning insulating at $U_{c 2}$. PG refers to a pseudogap state and the metal-insulator transition line separates regions with opposite signs of $d \rho / d T$. The right panel highlights the "re-entrant" feature in the low-energy DOS with increasing temperature. To avoid clutter we have not marked the $U_{P G}$ scale in the ground state in the left figure.

A comment about the magnetic state. Since there was no reason to expect that the moments would have any obvious periodic pattern, the only way to do the "minimization" was via simulated annealing employing Monte Carlo. In the $U / t \rightarrow \infty$ limit the half-filled Hubbard model leads to a Heisenberg model for the $\mathbf{m}_{i}$ (classical, in our SPA approximation) and for the pyrochlore Heisenberg antiferromagnet the moments are known to be disordered [10,11], albeit power-law correlated $[23,24]$. Our MC minimization reproduces this state. At lower $U / t$, both in the Mott phase and the "metal", there are no simple results known-but our results suggest that a "disordered" state persists and correlations reminiscent of a classical spin-liquid phase [23] survive down to $U / t \sim 8$.

\section{B. Thermal phase diagram}

Figure 2 (left panel) shows the $U-T$ phase diagram in terms of the magnetic, transport, and spectral properties that we observe. The following features emerge:

At finite $T$ thermal fluctuations of local moments on the weak-coupling side $\left(U<U_{P G}\right)$ lead to a quick low- $T$ increase in the low-energy DOS, and then a gradual decrease with further increase in $T$. On the strong-coupling side $\left(U>U_{c 2}\right)$ the angular fluctuations of the local moments result in a slight smearing of the Mott gap with temperature and an increase in the low-energy DOS. However, in the Mott-transition neighborhood, the Mott gap quickly converts to a PG with increasing $T$, leading to the widening of the $\mathrm{PG}$ region shown in Fig. 2 (left panel).

We demarcate the finite- $T$ metal-insulator boundary in terms of the temperature derivative $d \rho / d T$. A state is metallic if $d \rho / d T>0$ and insulating if $d \rho / d T<0$. The spectral features and resistivity are discussed in detail further on.

Figure 2 (right panel) shows the DOS at the Fermi level varying with $U$ and $T$. On the Mott-insulating side $(U \geqslant$ $U_{c 2}$ ) we observe the DOS slowly increasing with temperature, which can be understood as the filling of the Mott gap. On the metallic side $\left(U_{c 1}<U<U_{c 2}\right)$ we see a nonmonotonic behavior. The DOS quickly grows with temperature in the low-temperature regime. It then reduces with further increase in temperature, the weight getting transferred to high energy.

\section{Density of states}

Figure 3 shows the thermal evolution of the DOS in three of the four broad interaction regimes of our phase diagram.

(i) For $U<U_{c 1}$ the ground state is characterized by $m_{i}=\left|\mathbf{m}_{i}\right|=0$. The electron model reduces to the usual tightbinding pyrochlore lattice. This is characterized by two flat bands at the upper band edge and vanishing DOS at the Fermi energy. At finite $T$, small "randomly" oriented local moments appear in the system broadening the flat bands and leading to a small DOS at the Fermi level. (ii) For $U_{c 1}<U<U_{P G}$, the ground state has small disordered local moments. The DOS is gapless and the weight at the Fermi level is nonmonotonic with $T$, increasing initially and then decreasing as a weak PG forms [see Fig. 3(a)]. (iii) For $U_{P G}<U<U_{c 2}$ the DOS has a PG at $T=0$. This fills up initially with increasing $T$ [Fig. 3(b)], but deepens again above a temperature scale that is visible in Fig. 2, right panel. (iv) For $U \gg U_{c 2}$ the ground state has a hard gap. With increase in temperature, the angular fluctuations of the local moments result in a slight

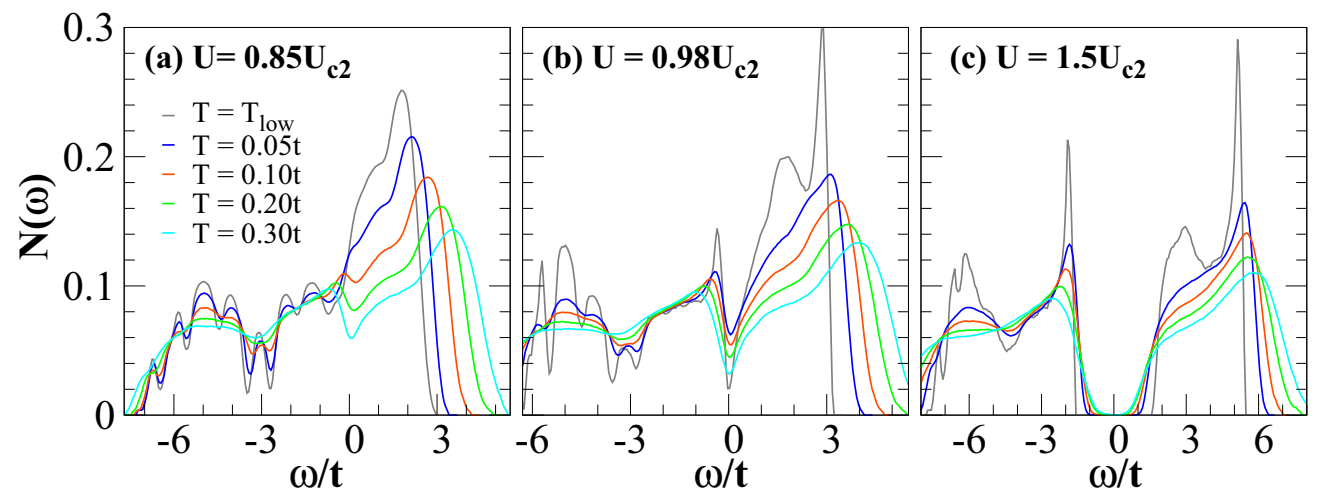

FIG. 3. Density of states varying with temperature at three representative regimes of our calculation. (a) $U=0.85 U_{c 2}$ lies in the gapless metallic side, (b) $U=0.98 U_{c 2}$ corresponds to the pseudogap (PG) regime with a pronounced dip in the DOS at the Fermi-level, and (c) $U=1.5 U_{c 2}$ lies in the gapped Mott-insulating side. $T_{\text {low }}=0.01 t$ in panel (a), whereas $T_{\text {low }}=0.0$ for panels (b) and (c). In panel (a) the low-energy DOS reduces with increasing $T$, in panel (b) it increases and then reduces with $T$, and in panel (c) it monotonically increases with $T$. 


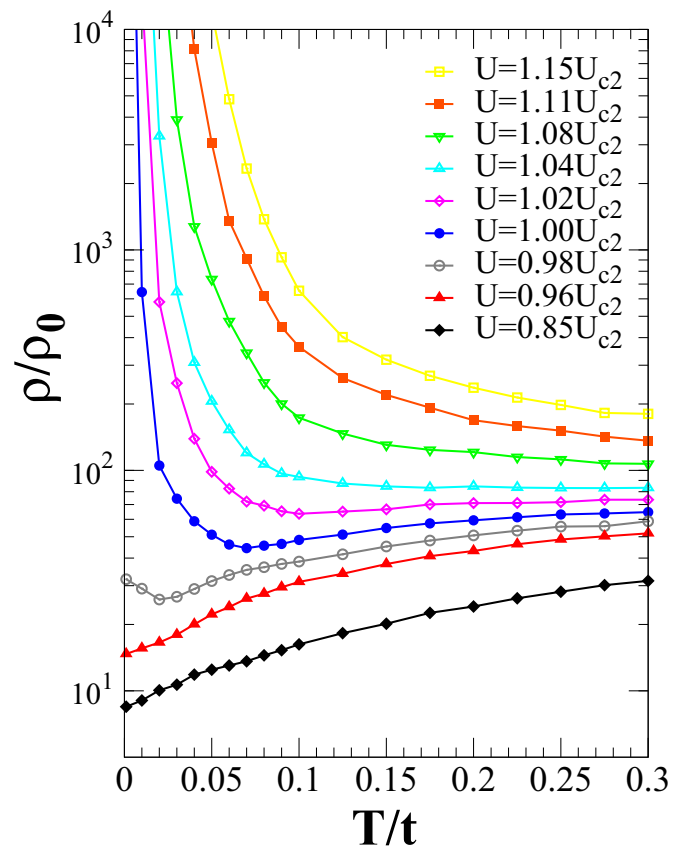

FIG. 4. Temperature dependence of the resistivity for different $U / t$ near the Mott insulator-metal transition. The normalizing scale is $\rho_{0}=\hbar / e^{2}$.

smearing of the Mott gap, and an increase in the low-energy DOS [Fig. 3(c)]. However, there exists a clear Mott gap until very high temperature, $T \sim m_{\mathrm{avg}} U$.

\section{Transport and optics}

Figure 4 shows the dc resistivity $\rho(T)$ for different $U / t$. We consider the four regimes. (i) For $U<U_{c 1}$ the $T=0$ phase is a semimetal. Since this lies well below the Mott transition we do not show the $T$ dependence here. (ii) For $U_{c 1}<U<$ $U_{P G}$ the residual resistivity $\rho(0)$ is finite with $d \rho / d T>0$ over the entire $T$ range. The resistivity can be understood in terms of a disorder-induced density of states and the scattering of electrons from the small disordered moments. This is the metallic regime. (iii) For $U \gg U_{c 2}$ the system has a clear Mott gap at $T=0$ with $\rho(0) \rightarrow \infty$. In this regime $d \rho / d T<0$ over the entire temperature window. This is the Mott-insulating regime. (iv) In the neighborhood of $U_{c 2}$, i.e., $\left|U-U_{c 2}\right| \ll U_{c 2}$, $\rho(T)$ shows a nonmonotonic behavior. We observe $d \rho / d T<0$ in the low temperature limit, crossing over to $d \rho / d T>0$ with increasing $T$. The temperature at which $d \rho / d T$ changes its sign is indicated as the $T_{M I T}$.

We observe $T_{M I T}$ increasing with $U$, as seen in Fig. 2. This behavior can be understood as the scattering of electrons from the background fluctuating local moments. As $U$ increases, the average local-moment magnitude $m_{\text {avg }}(U)$ also increases, resulting in the increased scattering of the electrons and a depleting DOS at the Fermi level.

Figure 5 shows the optical conductivity from our calculation as we cross the Mott transition. The important points are as follows: (i) $\sigma(\omega)$ for $U<U_{c 1}$ is a semimetal at $T=0$ and does not have a Drude peak. (ii) For $U_{c 1}<U<U_{P G}, \sigma(\omega)$ shows a response with the peak at a small finite frequency that slowly shifts to higher values with increasing $T$. (iii) For $U>U_{c 2}$ the system has a clear gap $\Delta(T)$ in the DOS. Thus $\sigma(\omega)=0$ for $\omega<\omega_{c} \sim \Delta(T)$. With increasing temperature, the gap $\Delta(T)$ reduces, resulting in small but increasing low-frequency weight of $\sigma(\omega)$ and the peak position shifts to higher frequency. This Mott-insulating regime of the pyrochlore lattice may have finite spectral weight at $\omega=0$ in the optical conductivity $\sigma(\omega)$ at high temperatures. (iv) For $U_{P G}<U<U_{c 2}$ we have a pseudogap in the DOS. $\sigma(\omega=0) \rightarrow 0$ in this regime. However, with increasing temperature the zero-frequency weight increases initially and then decreases in accord with the behavior of the DOS in this regime.

\section{DISCUSSION}

\section{A. Overall scenario}

Within our picture, the interaction effects are encoded in the presence of the "local moments" $\mathbf{m}_{i}$. The size $m_{i}$ of this moment dictates the onsite splitting at the site $\mathbf{R}_{i}$ and leads to a Mott gap in the overall DOS when $U m_{\mathrm{avg}} \gg t$. The spatial correlations of $\mathbf{m}_{i}$ decide whether the electron spin $\vec{\sigma}_{i}$ will display any long-range order.

Let us correlate the electron physics across the Mott transition to the behavior of the $\mathbf{m}_{i}$, we will then take up

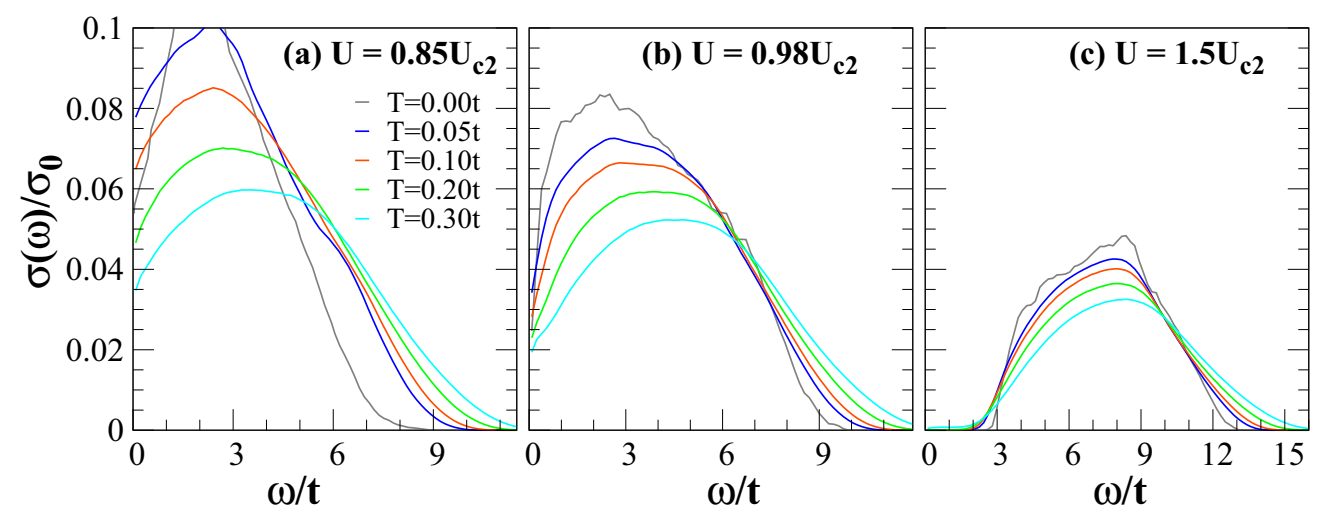

FIG. 5. Optical conductivity at $U / U_{c 2}=0.85,0.98$, and 1.5 with varying temperatures. $U=0.85 U_{c 2}$ shows a non-Drude-like behavior with peak at small and finite frequency. With increasing $U$ the peak moves to higher frequency and the zero-frequency weight decreases continuously and eventually there appears a gap for $U \geqslant U_{c 2}$. 


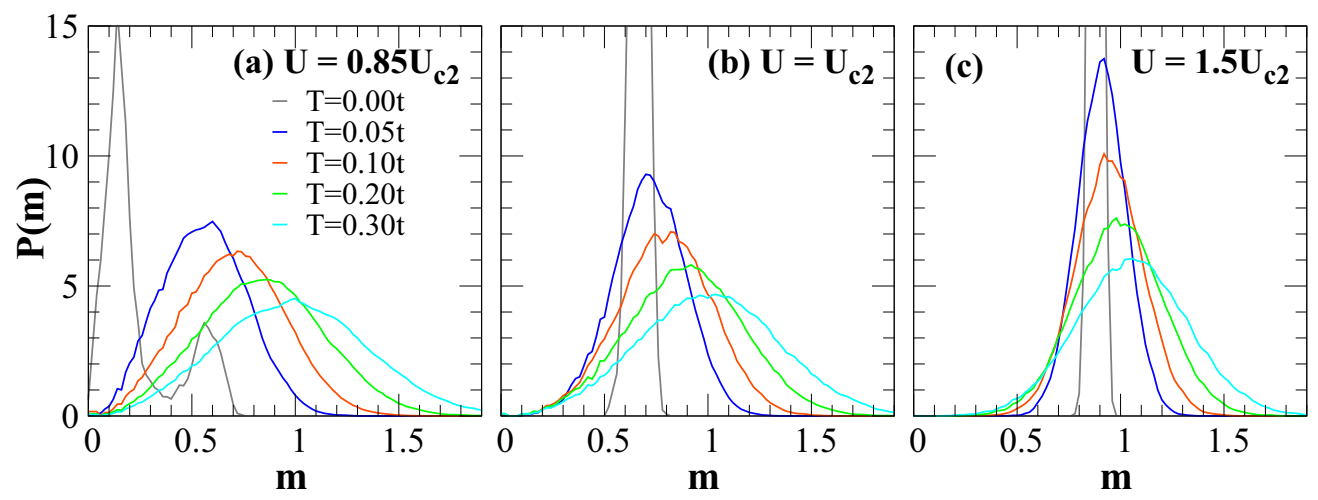

FIG. 6. Temperature dependence of $P(m)$ for $U=0.85 U_{c 2}, U_{c 2}$, and $1.5 U_{c 2}$ for indicated temperatures.

the effective models for the $\mathbf{m}_{i}$ themselves. (i) In the metallic regime, $U \gtrsim U_{c 1}$, the $m_{i}$ are small and the orientations are random. The scattering from these moments leads to a finite broadening $\tau_{\mathbf{k}}^{-1}$ of the momentum eigenstates. This generates a finite $\operatorname{DOS}$ at $\omega=0$, a non-Drude optical response, and resistivity increasing with temperature (unlike in a semimetal). (ii) In the Mott-insulating regime, $U>U_{c 2}$, the $m_{i}$ are large and show short-range magnetic correlation (discussed next). The large $m_{i}$ lead to a Mott gap in the DOS and optical conductivity, and a diverging resistivity as $T \rightarrow 0$. (iii) In the pseudogap regime, the $m_{i}$ are moderately large and orientationally disordered. This results in a strong suppression in the DOS at Fermi energy, but no gap, a large finite residual resistivity and a non-Drude optical response.

We have shown $m_{\text {avg }}$ for varying $U$ at $T=0$ [Fig. 1(b)]. In the next section we discuss the limiting models that dictate the behavior of $\mathbf{m}_{i}$, and in the section after, we show detailed results on the size distribution of $\mathbf{m}_{i}$, and its spatial correlations.

\section{B. Effective models in limiting cases}

We have shown only a formal expression for the effective magnetic Hamiltonian. To get a feel for the magnetic states that arise it is useful to provide the approximate analytic structure in limiting cases. These are (a) weak coupling, when $U \lesssim t$, and (b) strong coupling, when $U \gg t$.

\section{Weak coupling}

Our effective interaction looks like a "Hund's coupling", with the electron spin coupled to a background moment through the coupling $U$. Given the formal similarity with the Hund's problem we can borrow the form of the weak-coupling result [47] from the literature:

$$
\begin{aligned}
H_{\mathrm{eff}}\left\{\mathbf{m}_{i}\right\} \sim & -\frac{U^{2}}{4} \sum_{i j}\left(\chi_{i j}-\frac{1}{U} \delta_{i j}\right) \mathbf{m}_{i} \cdot \mathbf{m}_{j} \\
& +\frac{U^{4}}{16} \sum_{i j}^{k l} f\left(\mathbf{m}_{i}, \mathbf{m}_{j}, \mathbf{m}_{k}, \mathbf{m}_{l}\right)+\cdots .
\end{aligned}
$$

The structure of $f\left(\mathbf{m}_{i}, \ldots, \mathbf{m}_{l}\right)$ is complicated, involving two-spin terms such as $\mathbf{m}_{i} \cdot \mathbf{m}_{j},\left(\mathbf{m}_{i} \cdot \mathbf{m}_{j}\right)^{2}$, three-spin terms such as $\left(\mathbf{m}_{i} \cdot \mathbf{m}_{j}\right)\left(\mathbf{m}_{i} \cdot \mathbf{m}_{k}\right)$, and four-spin terms such as $\left(\mathbf{m}_{i} \cdot \mathbf{m}_{j}\right)\left(\mathbf{m}_{k} \cdot \mathbf{m}_{l}\right)$. The $i, j, k, l$ can be separated by long distance in the weak-coupling limit.

In contrast to Hund's problem, where there were predefined local moments, in our case the moments have to arise from an instability in the electron system. The leading instability involves the vanishing coefficient of the quadratic term, i.e., $1-U \chi_{0}(\mathbf{q})=0$. This generates the moment and, if there is a prominent peak at some $\mathbf{q}=\mathbf{Q}$ in $\chi_{0}$, the moments order with that wave vector. In such a situation the fourth-order term can be expanded about $\mathbf{Q}$. The quartic term decides the magnitude of the order at $\mathbf{Q}$.

We have computed the $\chi_{0}(\mathbf{q})$ for the half-filled pyrochlore band, having tested the scheme for the quarter-filled band for which results are available [48]. The $\chi_{0}(\mathbf{q})$ is featureless, suggesting that there is no particular wave vector that would be picked out. In that case the quartic term, whose detailed structure we do not know at half filling, determines not only the magnitude but also the spatial character of the order parameter field. It seems that the nonlinearity creates a bimodal distribution for the $m_{i}$, discussed further on, but without any significant spatial correlation.

\section{Strong coupling}

For $U \gg t$, one can write an effective magnetic Hamiltonian on the pyrochlore lattice by tracing out fermions order by order in $t / U$. This gives

$$
\begin{aligned}
H_{\mathrm{eff}}\{\mathbf{m}\} & =H_{\mathrm{tetr}}\{\mathbf{m}\}+H_{\text {coup }}\{\mathbf{m}\}, \\
H_{\text {tetr }}\{\mathbf{m}\} & \sim \sum_{\alpha}\left(J_{0}+J_{2} \mathbf{P}_{\alpha}^{2}+J_{4} \mathbf{P}_{\alpha}^{4}+\cdots\right), \\
H_{\text {coup }}\{\mathbf{m}\} & \sim J_{4}^{\prime} \sum_{i \in \alpha, j \in \beta} \mathbf{S}_{i} \cdot \mathbf{S}_{j}+J_{4}^{\prime \prime} \sum_{i \in \alpha, j \in \beta}^{k \in \alpha \cap \beta}\left(\mathbf{S}_{i} \cdot \mathbf{S}_{k}\right)\left(\mathbf{S}_{j} \cdot \mathbf{S}_{k}\right) \cdots,
\end{aligned}
$$

where $\mathbf{P}_{\alpha}=\sum_{i=1}^{4} \mathbf{m}_{i}^{\alpha}$ is the total spin on the tetrahedron $\alpha$, $H_{\text {tetr }}$ describes interactions between spins in a tetrahedron while $H_{\text {coup }}$ includes the intertetrahedron terms with a common corner-shared site. $J_{0}=-\frac{8 t^{2}}{U}\left(1-\frac{4 t^{2}}{U^{2}}\right), \quad J_{2}=\frac{t^{2}}{2 U}\left(1-\frac{24 t^{2}}{U^{2}}\right)$, $J_{4}=\frac{5 t^{4}}{8 U^{3}}$, and $J_{4}^{\prime}, J_{4}^{\prime \prime} \sim O\left(\frac{t^{4}}{U^{3}}\right)$.

Deep in the Mott phase, one would drop the $J_{4}, J_{4}^{\prime}$, and $J_{4}^{\prime \prime}$ terms and obtain a classical Heisenberg model, which shows no long-range order or freezing, but power-law correlations at low temperature $[23,24]$. The correlations of the electron 

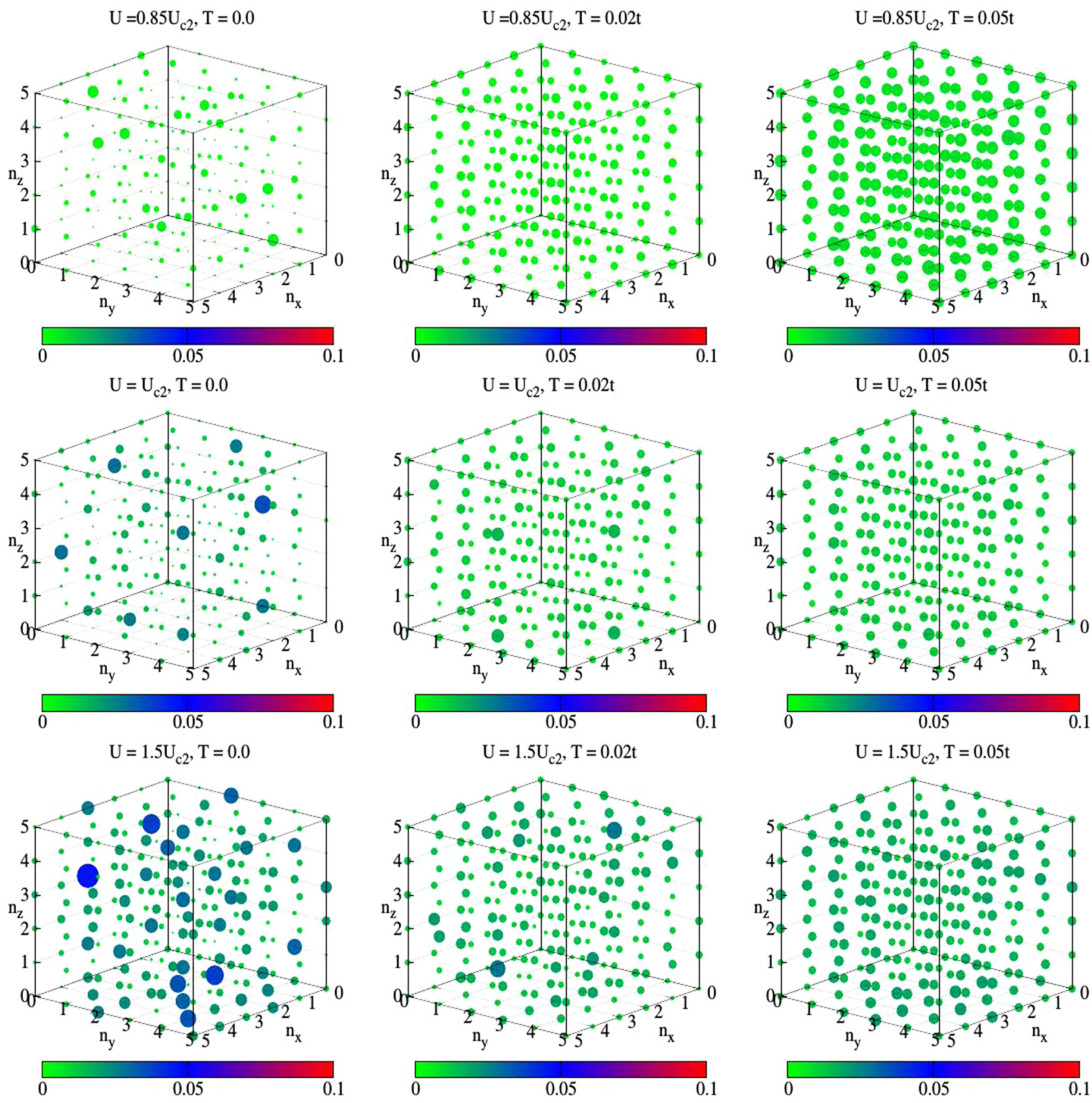

FIG. 7. The full magnetic structure factor $S(\mathbf{q})$ for $U=0.85 U_{c 2}, U_{c 2}, 1.5 U_{c 2}$ (along column) and $T=0.0,0.02 t$, and $0.05 t$ (along row). We use the notation $\mathbf{q}=\frac{2 \pi}{L}\left(n_{x}, n_{y}, n_{z}\right)$, where $n_{i}$ are integers. The size of a dot signifies relative weight at a given $\mathbf{q}$ while its color represents the actual magnitude of $S(\mathbf{q})$.

spins, $\vec{\sigma}_{i}$, can be computed in response to this. As $U \rightarrow U_{c 2}$ the $J_{4}, J_{4}^{\prime}$, and $J_{4}^{\prime \prime}$ terms become important. These multispin exchange interactions modify the magnetic ground state, but some of the Heisenberg-limit features [23] are observable (see next) in the structure factor down to $U \sim 8 t$. The expansion in $t / U$ ceases to be useful once the gap closes.

\section{Detailed magnetic structure}

Figure 6 shows the the amplitude distribution $P(m)$ of the magnetic moment for different $T$ and interaction regimes.
Figure 6(a) shows $P(m)$ for $U=0.85 U_{c 2}$. In the ground state there is a two-peak structure, highlighting the presence of amplitude inhomogeneity. We have checked that there is no significant density inhomogeneity or charge ordering in the system. With rise in temperature, $P(m)$ shows a broad singlepeak behavior with the peak shifting towards large $m$. This behavior is seen in the $U_{c 1}<U<U_{c 2}$ regime. Figure 6(b) shows $P(m)$ for $U \sim U_{c 2}$, just in the Mott-insulating side. The ground state has a narrow single-peak feature indicating an amplitude-homogeneous Mott state. With rise in temperature 
this narrow peak broadens, and the peak position moves towards higher $m$. Figure 6(c) shows $P(m)$ for $U=1.5 U_{c 2}$, well in the Mott regime. $P(m)$ has a single peak feature which broadens with temperature and shifts to higher $m$. The fluctuations about the mean are weaker in the insulator than in the metallic state.

Figure 7 shows the $\mathbf{q}$ dependence of the magnetic structure factor for varying $T$ and $U$. We observe that, even at $T=0, S(\mathbf{q})$ has no ordering peak at any $\mathbf{q}$. The magnetic ground state is disordered. Nevertheless, for both $U \sim U_{c 2}$ and $U=1.5 U_{c 2}$ the weight distribution is not completely homogeneous in $\mathbf{q}$ and has some prominent features. This signature survives to $T \sim 0.03 t$. Examination of the classical Heisenberg model has revealed that there are pinch-point features in scattering, arising from the constrained ground state, that survive [49] to $T \sim 0.1 J$, where $J$ is the exchange scale (in our case $J=t^{2} / U$ ). Obtaining such a result, indicative of power law correlations, requires larger system size $\left(\gtrsim 10^{3}\right)$, much longer annealing ( $\gtrsim 10^{8} \mathrm{MC}$ sweeps), and a more sophisticated algorithm instead of a single spin update. Due to our computational cost and size limitations we only get a hint of this spin-liquid state. By the time $T \sim 0.05 t$, which is $\sim J / 2$ at $U=1.5 U_{c 2}$, the q dependence is completely featureless.

\section{Methodological issues}

There are several approximations, analytic and numerical, we had to make to achieve some headway. Let us comment on these one by one, and their effect on the reliability of our results.

\section{Static approximation in Monte Carlo}

In principle, both spatial and temporal fluctuations could be important near the Mott transition on the pyrochlore lattice. However, fully handling the temporal fluctuations of the $\mathbf{m}_{i}$ and $\phi_{i}$ fields requires a quantum Monte Carlo scheme. The absence of such results is probably due to the sign problem for fermions in the frustrated geometry. We have retained only the zero Matsubara frequency, $\Omega_{n}=0$, mode of the auxiliary fields, exactly.

It is possible, but non trivial, to set up a Gaussian expansion for the finite $\Omega_{n}$ modes of $\mathbf{m}_{i}$ and $\phi_{i}$, while retaining and treating the $\Omega_{n}=0$ mode exactly as we have. This is a project for the future. In the absence of such a calculation we can only make the following conjectures:

(i) Local moments would remain well defined in the insulating phase, due to the gap. Since the present theory itself predicts no order at any $U$ or temperature, we do not expect additional quantum fluctuations to qualitatively modify the magnetic state.

(ii) A possible qualitative consequence of quantum fluctuations could be the restoration of translation invariance in the "magnetic-metal" phase. We cannot make a definite comment on this but, even if this were to happen, making the metallic state perfectly conducting at $T=0$, we believe that, above a low coherence temperature, one would see the signature of a highly resistive metal.

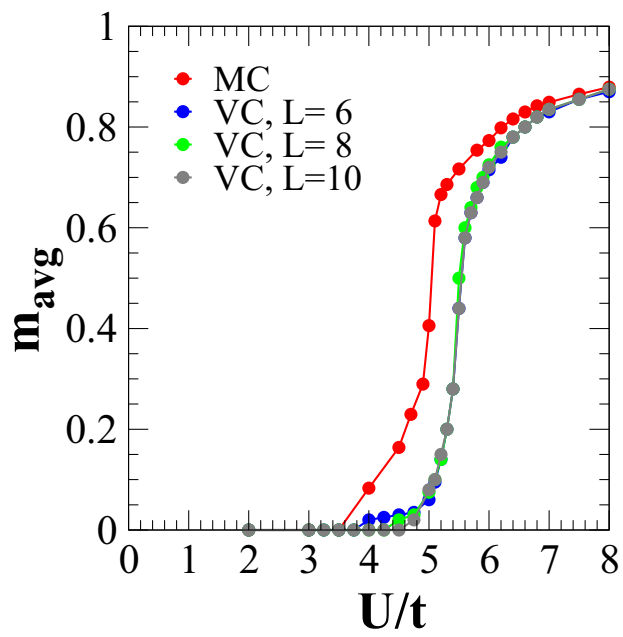

FIG. 8. Comparison of the variationally obtained averagemoment value with result from the Monte Carlo as $T \rightarrow 0$.

\section{Finite-size effects}

We have done our calculations on lattices that involve $\sim 800$ atoms. This is much larger than what is typically accessible in full-fledged fermion quantum Monte Carlo (QMC) (since temporal fluctuations also have to be included), allowing us to access resistivity and spectral features which do not involve artificial broadening. It is possible to try the calculations on $8^{3} \times 4$ lattices (involving about 2000 atoms) and checks at individual parameters do not reveal a significant difference.

\section{Cluster-based update}

Our Metropolis update involves a small cluster rather than diagonalization of the full Hamiltonian. This is well controlled in the large- $U$ limit when the "range" of electron excursion is limited but less reliable near the Mott transition. For that purpose we used a variational calculation, described below, that uses the MC result as an ansatz and checks the stability of such a state on large (up to $10^{3} \times 4$ lattices) for the ground state. The results are discussed below-and are qualitatively consistent with the cluster-based MC.

\section{Variational check}

Since MC hints that the magnetic ground state involves disordered local moments for $U>U_{c 1}$, we tried a simple variational check. We set up trial configurations $\left\{\mathbf{m}_{i}\right\}$ with random orientation, but uniform magnitude $m_{0}$, with $m_{0}$ as a variational parameter. This differs from the real situation where the $\mathbf{m}_{i}$ have some amplitude inhomogeneity and also orientational correlation. Energy minimization confirms the presence of a small moment phase, beyond some $U_{\text {low }}$, with an initial slow growth of $m_{0}(U)$ and then a rapid crossover to large values at some $U_{\text {high }}$ (see Fig. 8). The $U_{\text {low }}$ and $U_{\text {high }}$ are about $10 \%$ higher than MC estimates and may get reduced if spatial correlations are included.

\section{E. Relation to experiments}

Pyrochlore Mott materials include the rare-earth-based molybdates $R_{2} \mathrm{Mo}_{2} \mathrm{O}_{7}$ and iridates $R_{2} \mathrm{Ir}_{2} \mathrm{O}_{7}$ ( $R$ is a rare-earth 
TABLE I. Comparison of features of the pyrochlore Hubbard model with the models relevant for the molybdates and iridates.

\begin{tabular}{|c|c|c|c|}
\hline Indicator & Present theory & Molybdate & Iridate \\
\hline Degrees of freedom & One orbital per site & $\begin{array}{l}\text { Two orbitals per site } \\
\text { and a } S=1 / 2 \text { moment }\end{array}$ & $\begin{array}{l}\text { Effective one orbital } \\
\text { per site }\end{array}$ \\
\hline Interactions & Repulsive Hubbard & $\begin{array}{l}\text { Repulsive Hubbard, Hund's coupling, } \\
\text { antiferro superexchange }\end{array}$ & $\begin{array}{l}\text { Repulsive Hubbard, } \\
\text { spin-orbit coupling }\end{array}$ \\
\hline Weak-coupling phase & $\begin{array}{l}\text { Band semimetal, } \\
\text { spin-disordered metal }\end{array}$ & $\begin{array}{l}\text { Ferromagnetic metal, } \\
\text { spin-glass metal }\end{array}$ & Correlated metal \\
\hline Strong-coupling phase & Spin-liquid Mott insulator & Spin-glass Mott insulator & All-in-all-out Mott insulator \\
\hline Features in resistivity & $\begin{array}{l}\text { Continuous divergence } \\
\text { of } \rho(0) \text { with } U / t\end{array}$ & $\begin{array}{l}\text { Seemingly continuous divergence } \\
\text { of } \rho(T=0) \text { with } r_{R}\end{array}$ & $\begin{array}{l}\text { Seemingly continuous divergence } \\
\text { of } \rho(T=0) \text { with } r_{R}\end{array}$ \\
\hline $\begin{array}{l}\text { Magnetoresistance in } \\
\text { moderate field }\end{array}$ & Weak & $\begin{array}{l}\text { Huge: drives insulator-metal } \\
\text { transition }\left(\mathrm{Gd}_{2} \mathrm{Mo}_{2} \mathrm{O}_{7}\right)\end{array}$ & Weak \\
\hline Anomalous Hall response & Not explored & Observed in $\mathrm{Nd}_{2} \mathrm{Mo}_{2} \mathrm{O}_{7}$ & Observed in $\mathrm{Nd}_{2} \mathrm{Ir}_{2} \mathrm{O}_{7}$ \\
\hline
\end{tabular}

ion or Y). The Mo and Ir ions live on a pyrochlore lattice while the $R$ inhabit an interpenetrating pyrochlore structure.

In contrast to $3 d$-electron-based systems which are dominated by the Hubbard $U$, the molybdates involve $4 d$ electrons-where Hund's coupling $J_{H}$ is also important, while the iridates involve $5 d$ electrons-with spin-orbit coupling playing a vital role. In this paper we focus on the simple Hubbard model as the starting problem. In what follows we quickly list out the key molybdate and iridate Mott features (see Table I) and argue how some of these are already visible in our present results.

The molybdates exhibit a MIT with decreasing rare-earth ionic radius $r_{A}$. Materials with larger $r_{A}$ are ferromagnetic (FM) metals, those with small $r_{A}$ are spin-glass (SG) insulators $[12,13]$, and there is a SG metal phase [16] near the MIT. Pressure and magnetic field can drive an IMT in materials like $\mathrm{Gd}_{2} \mathrm{Mo}_{2} \mathrm{O}_{7}$ which are weakly insulating [17]. An anomalous Hall effect (AHE) has been observed [50-52] in $\mathrm{Nd}_{2} \mathrm{Mo}_{2} \mathrm{O}_{7}$ and is ascribed to nonvanishing spin chirality.

The iridates also show a MIT with decreasing $r_{A}$, but in this case the MI transition is accompanied by a magnetic transition from a paramagnetic to an antiferromagnetic allin-all-out (AIAO) ordering $[18,19,53]$. While the magnetic character differs distinctly from molybdates, iridates also show a pressure-driven insulator-metal transitions, via unusually resistive ground states [6,20,21], and spin-chirality-driven AHE [8] in materials like $\operatorname{Pr}_{2} \mathrm{Ir}_{2} \mathrm{O}_{7}$ [54].

Reducing $r_{A}$ reduces the hopping in these materials and is akin to increasing the $U / t$ ratio in our model. The general observation $[14,15,18,19]$ of a metal-insulator transition with reducing $r_{A}$ is consistent with our phase diagram. In the table above, however, we compare a set of indicators between molybdate or iridate experiments and our present theory. While the presence of electron correlation on a pyrochlore structure is common to all three, there are additional interactions present in the real materials. The one common feature that emerges is the occurrence of a high-resistivity ground state close to the MIT which, we believe, is due to the coupling of electrons to disordered spin and orbital moments. This is a general consequence of the interplay of correlation effects and strong geometric frustration. We will discuss the detailed iridate and molybdate theories elsewhere.

\section{CONCLUSION}

We have studied the Mott transition in a half-filled Hubbard model on a pyrochlore lattice. The geometric frustration and the corresponding large magnetic degeneracy prevents the occurrence of any magnetic order in the deep Mott state. This continues all the way to the insulator-metal transition. Beyond the insulator-metal transition there is a window with a pseudogap in the density of states, disordered local moments, and a large residual resistivity. At even weaker interaction one recovers the nonmagnetic-band semimetal. Thermal fluctuations destroy the "spin-liquid" correlations in the insulating state, converting the system to an uncorrelated paramagnet. The low-energy electronic density of states and the resistivity show a monotonic temperature dependence deep in the metallic and insulating phases, but a nonmonotonic character near the insulator-metal transition. A comparison with the rare-earth molybdates and iridates reveal a clear similarity in the resistivity, while detailed aspects of the magnetic state and magnetotransport are missed out. We are exploring these problems separately.

\section{ACKNOWLEDGMENTS}

We acknowledge use of the HPC clusters at HRI and a discussion with Shubhro Bhattacharjee. We thank Sauri Bhattacharyya for reading the manuscript. P.M. acknowledges support from an Outstanding Research Investigator grant of the Department of Atomic Energy-Science Research Council (DAE-SRC) of India.
[1] N. P. Ong and R. J. Cava, Science 305, 52 (2005).

[2] L. Balents, Nature (London) 464, 199 (2010).

[3] K. Kanoda, J. Phys. Soc. Jpn. 75, 051007 (2006); B. J. Powell and R. H. McKenzie, Rep. Prog. Phys. 74, 056501 (2011).
[4] Y. Imai, N. Kawakami, and H. Tsunetsugu, Phys. Rev. B 68, 195103 (2003); N. Bulut, W. Koshibae, and S. Maekawa, Phys. Rev. Lett. 95, 037001 (2005). 
[5] M. M. Abd-Elmeguid, B. Ni, D. I. Khomskii, R. Pocha, D. Johrendt, X. Wang, and K. Syassen, Phys. Rev. Lett. 93, 126403 (2004).

[6] K. Ueda, J. Fujioka, Y. Takahashi, T. Suzuki, S. Ishiwata, Y. Taguchi, and Y. Tokura, Phys. Rev. Lett. 109, 136402 (2012).

[7] G.-W. Chern, S. Maiti, and R. M. Fernandes, and P. Wolfle, Phys. Rev. Lett. 110, 146602 (2013).

[8] Y. Machida, S. Nakatsuji, Y. Maeno, T. Tayama, T. Sakakibara, and S. Onoda, Phys. Rev. Lett. 98, 057203 (2007).

[9] J. S. Gardner. M. J. P. Gingras, and J. E. Greedan, Rev. Mod. Phys. 82, 53 (2010).

[10] J. N. Reimers, Phys. Rev. B 45, 7287 (1992).

[11] R. Moessner and J. T. Chalker, Phys. Rev. B 58, 12049 (1998).

[12] B. D. Gaulin, J. N. Reimers, T. E. Mason, J. E. Greedan, and Z. Tun, Phys. Rev. Lett. 69, 3244 (1992).

[13] M. J. P. Gingras, C. V. Stager, N. P. Raju, B. D. Gaulin, and J. E. Greedan, Phys. Rev. Lett. 78, 947 (1997).

[14] Y. Taguchi and Y. Tokura, Phys. Rev. B 60, 10280 (1999).

[15] I. Kezsmarki, N. Hanasaki, K. Watanabe, S. Iguchi, Y. Taguchi, S. Miyasaka, and Y. Tokura, Phys. Rev. B 73, 125122 (2006).

[16] S. Iguchi, N. Hanasaki, M. Kinuhara, N. Takeshita, C. Terakura, Y. Taguchi, H. Takagi, and Y. Tokura, Phys. Rev. Lett. 102, 136407 (2009).

[17] N. Hanasaki, M. Kinuhara, I. Kezsmarki, S. Iguchi, S. Miyasaka, N. Takeshita, C. Terakura, H. Takagi, and Y. Tokura, Phys. Rev. Lett. 96, 116403 (2006).

[18] D. Yanagishima and Y. Maeno, J. Phys. Soc. Jpn. 70, 2880 (2001).

[19] K. Matsuhira, M. Wakeshima, Y. Hinatsu, and S. Takagi, J. Phys. Soc. Jpn. 80, 094701 (2011).

[20] M. Sakata, T. Kagayama, K. Shimizu, K. Matsuhira, S. Takagi, M. Wakeshima, and Y. Hinatsu, Phys. Rev. B 83, 041102 (2011).

[21] F. F. Tafti, J. J. Ishikawa, A. McCollam, S. Nakatsuji, and S. R. Julian, Phys. Rev. B 85, 205104 (2012).

[22] M. P. Zinkin, M. J. Harris, and T. Zeiske, Phys. Rev. B 56, 11786 (1997).

[23] C. L. Henley, Phys. Rev. B 71, 014424 (2005).

[24] S. V. Isakov, K. Gregor, R. Moessner, and S. L. Sondhi, Phys. Rev. Lett. 93, 167204 (2004).

[25] C. L. Henley, Phys. Rev. Lett. 96, 047201 (2006); U. Hizi and C. L. Henley, Phys. Rev. B 73, 054403 (2006).

[26] U. Hizi and C. L. Henley, Phys. Rev. B 80, 014407 (2009).

[27] B. Canals and C. Lacroix, Phys. Rev. Lett. 80, 2933 (1998).

[28] M. Hermele, M. P. A. Fisher, and L. Balents, Phys. Rev. B 69, 064404 (2004).

[29] B. Normand and Z. Nussinov, Phys. Rev. Lett. 112, 207202 (2014).
[30] D. Tsuneishi and H. Kawamura, J. Phys.: Condens. Matter 19, 145273 (2007).

[31] G.-W. Chern and R. Moessner, and O. Tchernyshyov, Phys. Rev. B 78, 144418 (2008).

[32] T. Okubo and T. H. Nguyen, and H. Kawamura, Phys. Rev. B 84, 144432 (2011).

[33] B. Javanparast, Z. Hao, and M. Enjalran, and M. J. P. Gingras, Phys. Rev. Lett. 114, 130601 (2015).

[34] S. Fujimoto, Phys. Rev. B 64, 085102 (2001); 67, 235102 (2003).

[35] W. Witczak-Krempa, A. Go, and Y. B. Kim, Phys. Rev. B 87, 155101 (2013).

[36] A. Go, W. Witczak-Krempa, G. S. Jeon, and K. Park, and Y. B. Kim, Phys. Rev. Lett. 109, 066401 (2012).

[37] I. V. Solovyev, Phys. Rev. B 67, 174406 (2003).

[38] Y. Motome and N. Furukawa, J. Phys.: Conf. Ser. 320, 012060 (2011).

[39] J. Hubbard, Phys. Rev. Lett. 3, 77 (1959).

[40] J. Hubbard, Phys. Rev. B 19, 2626 (1979).

[41] H. J. Schulz, Phys. Rev. Lett. 65, 2462 (1990).

[42] M. Mayr, G. Alvarez, C. Sen, and E. Dagotto, Phys. Rev. Lett. 94, 217001 (2005).

[43] Y. Dubi, Y. Meir, and Y. Avishai, Nature (London) 449, 876 (2007).

[44] R. Tiwari and P. Majumdar, Europhys. Lett. 108, 27007 (2014).

[45] S. Kumar and P. Majumdar, Eur. Phys. J. B 50, 571 (2006).

[46] P. B. Allen, in Conceptual Foundation of Materials, edited by S. G. Louie and M. L. Cohen (Elsevier, Amsterdam, 2006), Vol. 2.

[47] Y. Akagi, M. Udagawa, and Y. Motome, Phys. Rev. Lett. 108, 096401 (2012).

[48] G.-W. Chern, Phys. Rev. Lett. 105, 226403 (2010).

[49] P. H. Conlon and J. T. Chalker, Phys. Rev. B 81, 224413 (2010).

[50] T. Katsufuji, H. Y. Hwang, and S.-W. Cheong, Phys. Rev. Lett. 84, 1998 (2000).

[51] Y. Taguchi, T. Sasaki, S. Awaji, Y. Iwasa, T. Tayama, T. Sakakibara, S. Iguchi, T. Ito, and Y. Tokura, Phys. Rev. Lett. 90, 257202 (2003).

[52] K. Ueda, S. Iguchi, T. Suzuki, S. Ishiwata, Y. Taguchi, and Y. Tokura, Phys. Rev. Lett. 108, 156601 (2012).

[53] W. Witczak-Krempa and Y. B. Kim, Phys. Rev. B 85, 045124 (2012); E. Kin-Ho Lee, S. Bhattacharjee, and Y. B. Kim, ibid. 87, 214416 (2013).

[54] S. Nakatsuji, Y. Machida, Y. Maeno, T. Tayama, T. Sakakibara, J. van Duijn, L. Balicas, J. N. Millican, R. T. Macaluso, and Julia Y. Chan, Phys. Rev. Lett. 96, 087204 (2006). 